Document downloaded from:

http://hdl.handle.net/10251/51228

This paper must be cited as:

Tung, MM.; Weinmüller, EB. (2013). Gravitational frequency shifts in transformation acoustics. EPL. 101(5):54006-54011. doi:10.1209/0295-5075/101/54006.

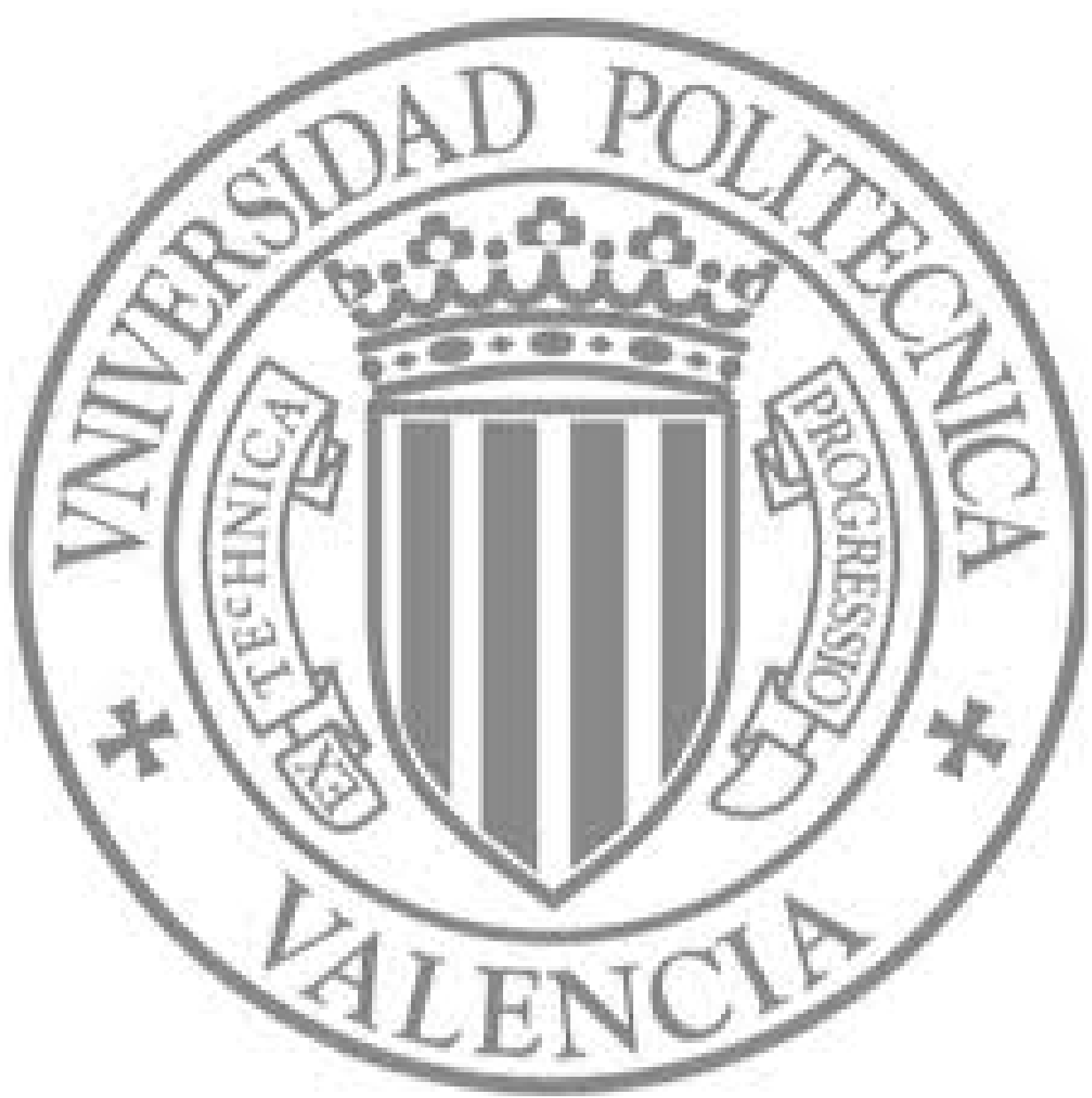

The final publication is available at

http://iopscience.iop.org/0295-5075/101/5/54006/

Copyright European Physical Society 


\title{
Gravitational frequency shifts in transformation acoustics
}

\author{
Michael M. TUnG ${ }^{1(a)}$ and EWA B. WeINMÜLLER ${ }^{2}$ \\ 1 Instituto de Matemática Multidisciplinar, Universitat Politècnica de València \\ Camino de Vera s/n, E-46022 Valencia, Spain, EU \\ 2 Institute for Analysis and Scientific Computing, Vienna University of Technology \\ Wiedner Hauptstrasse 8-10/1152, A-1040 Wien, Austria, EU
}

\author{
PACS $43.90 .+\mathrm{v}$ - Other topics in acoustics \\ PACS 03.65.Pm - Relativistic wave equations \\ PACS 04.20.Fy - Canonical formalism, Lagrangians, and variational principles
}

\begin{abstract}
In metamaterial acoustics, it is conceivable that any type of fine-tuned acoustic properties far beyond those found in nature may be transferred to an appropriate medium. Effective design and engineering of these modern acoustic metadevices poses one of the forefront challenges in this field. As a practical example of a new covariant approach for modelling acoustics on spacetime manifolds, we choose to implement the acoustic analogue of the frequency shift due to gravitational time-dilation. In accordance with Einstein's equivalence principle, two different spacetimes, corresponding to uniform acceleration or uniform gravity, are considered. For wave propagation in a uniformly accelerating rigid frame, an acoustic event horizon arises. The discussion includes a detailed numerical analysis for both spacetime geometries.
\end{abstract}

Introduction. - As the interdisciplinary science that studies the effects and properties of mechanical waves in various media, acoustics has come a long way from the initial attempts of the ancient Greek philosopher Pythagoras in the 6th century B.C. to explain the harmonic overtone series on a string up to the consolidation of the field of mathematical acoustics in the 19th century pioneered by Helmholtz and Rayleigh.

Recent successes in the design of optical analogue models of gravity, see, i.e., refs. [1-4], should also carry over to investigations for physical systems describing sound propagation in acoustic metamaterials. However, acoustic analogue models of gravity are so far little explored.

Acoustic metamaterials are mostly artificially produced with suitable and highly unusual acoustic properties that are not found in nature $[5,6]$. Advanced acoustic devices may be composed of these metamaterials, and their technical and industrial applications are numerous, ranging from the acoustic improvement of concert halls to the design of submarines undetectable for sonar probes. More interesting details on acoustic cloaking are found, for example, in ref. [6-8].

Clearly, the correspondence between transformation optics and transformation acoustics cannot be complete, as both field theories fundamentally differ in the underlying symmetry properties of their respective Lagrangians [9-12]. To fully describe electromagnetic phenomena, it is necessary to introduce

${ }^{(a)}$ E-mail: mtung@mat.upv.es a four-potential and additionally require that the theory possesses gauge invariance to reflect charge conservation, whereas the linear acoustic theory, as proposed in ref. [12], only relies on a scalar velocity potential and is scale-invariant due to the absence of any fixed length scale.

However, both theories allow for deformations of wave propagation space, which mathematically resembles spacetime of general relativity in the presence of gravitating matter or energy, so that the underlying mathematical structure is described by pseudo-Riemannian manifolds. As a consequence, transformation acoustics proposes to employ sophisticated differentialgeometric tools for the efficient design and manufacturing of these modern acoustic devices.

Recently, a theoretical Lagrangian framework was elaborated which generally describes the evolution of acoustic pressure through an appropriate metamedium [12]. It derives from a variational principle in fully covariant spacetime all the equations governing non-dissipative acoustic phenomena. Undoubtedly, this approach opens up many new practical possibilities for the construction of acoustic metadevices with unexpected characteristics.

In this letter, we present a novel application of transformation acoustics by implementing the gravitational frequencyshift analogue in acoustics, i.e., the acoustic imitation of a shift in radiation frequency due to a gravitational field. The measurement of gravitational frequency shifts provides some of the 
crucial experimental checks on the basic predictions of general relativity. It was in fact Einstein's famous elevator thought experiment, in which objects in a uniform gravitational field behave similarly to objects inside a uniformly accelerating frame, that led him to formulate his equivalence principle - the principle that would form the very foundation of general relativity.

A previous work centred on an optical analogue of the cosmological redshift [4]. Note, however, that the cosmological redshift is caused by the expansion of the universe and is therefore of dynamical origin. It should not be confused with the frequency shifts due to a static gravitational field, the subject of the present letter. These two types of frequency shifts are unrelated and based on entirely different spacetimes. Moreover, gravitational frequency shifts are a manifestation of waves travelling in a varying gravitational potential and as such a direct probe of the underlying relativistic theory of the gravitational field.

In the following, we will first introduce some necessary details of the framework describing spacetime for a reference frame with uniform acceleration or with a uniform gravitational field. Next, we proceed with the derivation of the corresponding acoustic wave equations in a most general manner via a variational principle. At the same time, we will also be able to derive the fundamental relations between the constitutive acoustic parameters, which provide the description on how to actually transfer the spacetime characteristics at hand into the acoustic metadevice. A numerical discussion will follow in order to visualize some of the metadevice's outstanding acoustic features.

Spacetime for uniform acceleration and gravity. - In this work, we intend to mimic the effect on the frequency of sound propagating in a suitable acoustic metamaterial which an accelerated observer detects, or alternatively, a gravitational field produces. For this purpose, we assume the following simple Euclidean form for the line element with static spacetime and the mixed signature $(-,+,+,+)$ :

$$
d s^{2}=-\alpha^{2}(y) c^{2} d t^{2}+d x^{2}+d y^{2}+d z^{2},
$$

where $c>0$ is the speed of sound within the medium. The line element is constructed in such a way that the gravitational field or the accelerated motion is aligned along the $y$-axis and the temporal scale factor $\alpha \neq 0$ only depends on the variable $y$. The underlying Lorentzian metric is then given by

$$
g_{\mu \nu}=\left(\begin{array}{cccc}
-\alpha^{2}(y) & 0 & 0 & 0 \\
0 & 1 & 0 & 0 \\
0 & 0 & 1 & 0 \\
0 & 0 & 0 & 1
\end{array}\right)
$$

Note that $\sqrt{-g}=|\alpha|$ holds, using the standard notation $g=$ $\operatorname{det}\left(g_{\mu \nu}\right)$ for the determinant of the metric.

For a uniformly accelerating frame (UAF) and a uniform gravitational field (UGF), Desloge proposed the following two scale factors [13-15]:

$$
\alpha(y)=\left\{\begin{array}{r}
1+\frac{g_{0}}{c^{2}} y \\
e^{\frac{g_{0}}{c^{2}} y} .
\end{array}\right.
$$

Here, $g_{0}$ is the constant acceleration related to the moving frame or the fixed strength of the gravitational field within the rest frame, respectively. Observe that for both cases the metric, eq. (2), does not converge to the Minkowski metric at spatial infinity. Moreover, the UAF metric is singular at $y=-c^{2} / g_{0}$, which corresponds to a typical Rindler event horizon.

It is not difficult to check that the only non-vanishing Christoffel symbols are

$$
\begin{aligned}
& \Gamma_{00}^{2}=\alpha(y) \alpha^{\prime}(y), \\
& \Gamma_{02}^{0}=\frac{\alpha^{\prime}(y)}{\alpha(y)} .
\end{aligned}
$$

Then, there exists one independent component of the Riemann curvature tensor, namely [13]

$$
R_{0220}=-\alpha(y) \alpha^{\prime \prime}(y),
$$

which demonstrates that the underlying UGF spacetime is not empty and is curved $\left(R_{0220} \neq 0\right)$, whereas the UAF spacetime is flat $\left(R_{0220}=0\right)$ and represents a pseudo-force. Furthermore, the components of the Einstein tensor are given by

$$
G_{11}=G_{33}=\frac{\alpha^{\prime \prime}(y)}{\alpha(y)}, \quad G_{00}=G_{22}=0,
$$

which shows that only the UAF metric is an exact solution of the vacuum Einstein equations.

In fact, eqs. (4) readily yield for the geodesics of the relevant $y$-direction (acceleration occurs only in this direction and thus $\ddot{x}=\ddot{z}=0$ ) the general formula

$$
\ddot{y}+c^{2} \alpha(y) \alpha^{\prime}(y)=0,
$$

which results in

$$
\ddot{y}=\left\{\begin{array}{l}
-g_{0}\left(1+\frac{g_{0}}{c^{2}} y\right), \\
-g_{0} e^{\frac{2 g_{0}}{c^{2}} y} .
\end{array}\right.
$$

These two expressions are approximately equal for sufficiently small values of $y$. In the regime $|y| \ll c^{2} / g_{0}$, observations made in the UAF frame are physically equivalent to those made in the UGF frame. Note also that under the exchange $y \leftrightarrow$ $-\left(y+2 c^{2} / g_{0}\right)$ the UAF acceleration in eq. (8) switches sign, which produces a change in direction at $y=-c^{2} / g_{0}$ and again is linked to the presence of the Rindler horizon separating the physical domains $y>-c^{2} / g_{0}$ and $y<-c^{2} / g_{0}$.

An interesting discussion on the physical relation between reference frames at rest with a uniform gravitational field and uniformly accelerating frames in field-free space in connection with the validity of the equivalence principle is provided in ref. [16]. For our means, it will suffice to accept both cases as viable models and testing ground for further investigations in transformation acoustics. 
Hamilton's principle and acoustic wave equation. - In the context of transformation acoustics, Hamilton's principle states that the evolution of a non-dissipative acoustic system is described by a variational principle for the generalized coordinate $\phi: M \rightarrow \mathbb{R}$, where $\phi$ denotes the scalar potential of the acoustic metafluid defined on the pseudo-Riemannian spacetime manifold $M$ with a smooth, symmetric metric $\boldsymbol{g}$ : $T_{p} M \times T_{p} M \rightarrow \mathbb{R}$. If the state of $\phi$ on the boundary $\partial \Omega$ of a bounded, closed set of spacetime $\Omega \subset M$ is known, then the source-free dynamics on $\Omega$ is fully determined by the requirement that the following functional derivative vanishes [12]:

$$
\frac{\delta}{\delta \phi} \int_{\Omega} d^{4} x\left(\frac{1}{2} \sqrt{-g} g^{\mu v} \phi_{, \mu} \phi_{, v}\right)=0,
$$

where the conventional comma notation in the index refers to a partial derivative of the respective spacetime component, and $g^{\mu v}$ is the inverse metric tensor in a particular coordinate frame. For the spacetime integration of eq. (9), the invariant volume element is as usual given by $d^{4} x \sqrt{-g}=d x^{0} d x^{1} d x^{2} d x^{3} \sqrt{-g}$ and the bracket just contains the kinetic term of the acoustic field in covariant four-dimensional form [12]. This formalism automatically guarantees the essential condition that the resulting acoustic wave equation will be invariant under certain coordinate transformations.

By substituting the metric under consideration, eq. (2), into the variational principle, eq. (9), we obtain

$$
\frac{\delta}{\delta \phi} \int_{\Omega} d^{4} x \frac{1}{2}|\alpha|\left[\nabla^{2} \phi-\frac{1}{c^{2} \alpha^{2}} \dot{\phi}\right]=0 .
$$

Using standard differential-geometric methods (see e.g. ref. [17]), one can directly derive the acoustic wave equation in curved spacetime for the spacetime metric at hand from the variation of the action, eq. (10). After some simplification and the suppression of the physically uninteresting third space component, this yields

$$
\begin{aligned}
& \Delta_{M} \phi=\frac{1}{\sqrt{-g}}\left(\sqrt{-g} g^{\mu \nu} \phi_{, \mu}\right)_{, \nu} \\
& =-\frac{1}{\alpha^{2}} \phi_{, 00}+\phi_{, 11}+\frac{1}{|\alpha|}\left(|\alpha| \phi_{, 2}\right)_{, 2}=0,
\end{aligned}
$$

where $\Delta_{M} \phi$ is the Laplace-Beltrami operator on manifold $M$. Note that eq. (11) completely determines the acoustic wave propagation in the prefabricated metamaterial under consideration. Every concrete physical spacetime geometry has its own acoustic equivalent which then may become subject to further investigation - in full agreement with the gravity analogue programme [18].

The relevant acoustic parameters are the scalar bulk modulus $\kappa: M \rightarrow \mathbb{R}_{+}$and the anisotropic mass-density tensor $\rho: T_{p} N \times T_{p} N \rightarrow \mathbb{R}$ on a smooth three-dimensional manifold $N$. We are now in the position to identify the fundamental constitutive relations between these parameters [12], linking together the virtual space (flat space with Minkowski metric $\bar{g}_{\mu \nu}=\operatorname{diag}(-1,1,1,1)$ and known wave propagation) and physical space (curved, transformed space with metric $g_{\mu \nu}$ and the desirable acoustic properties). Then, using $\sqrt{-\bar{g}}=1$ and $\sqrt{-g}=|\alpha|$ immediately gives

$$
\begin{aligned}
\kappa & =\frac{\sqrt{-g}}{\sqrt{-\bar{g}}} \bar{\kappa}=|\alpha(y)| \bar{\kappa}, \\
\rho_{0} \rho^{i j}= & \frac{\sqrt{-\bar{g}}}{\sqrt{-g}} \bar{g}^{i j}=\frac{1}{|\alpha(y)|}\left(\begin{array}{lll}
1 & 0 & 0 \\
0 & 1 & 0 \\
0 & 0 & 1
\end{array}\right),
\end{aligned}
$$

where $\rho_{0}$ denotes the metafluid density and $\rho^{i j}$ is the inverse of the density tensor $\rho$ in local coordinates. Obviously, the density tensor, eq. (12b), has the following, particularly simple form in a Euclidean frame:

$$
\boldsymbol{\rho}=\rho_{0}|\alpha(y)| \boldsymbol{I}_{3},
$$

where $\boldsymbol{I}_{3}$ is just the three-dimensional identity. This means, as expected, that the UAF and UGF acoustic systems display full isotropy, but contain a crucial change of scale in the $y$ direction, the direction to be chosen to align with the acceleration or force field, respectively. In summary, eqs. (12a) and (13) precisely provide the recipe for engineering the physical acoustic metamedia with Desloge's underlying spacetime metrics.

For a more detailed analysis of the wave propagation in such spacetime, we apply the method of separation of variables to the acoustic potential in eq. (11) by taking

$$
\phi(t, x, y)=\phi_{0}(t) \phi_{1}(x) \phi_{2}(y) \text {. }
$$

Then, for angular frequency $\omega$, a straightforward calculation gives the standard harmonic time-dependence of theoretical acoustics

$$
\phi_{0}(t)=e^{i \omega t}
$$

and the plane-wave solution for a progressive wave in the $x$ direction

$$
\phi_{1}(x)=A^{+} e^{-i k_{x} x}
$$

with corresponding wavenumber $k_{x}$ and amplitude $A^{+}$(see e.g. ref. [19]). The $y$-dependence is of main interest and dictated by the following differential equation

$$
\phi_{2}^{\prime \prime}+\frac{\alpha^{\prime}}{\alpha} \phi_{2}^{\prime}+\left(\frac{\omega^{2}}{c^{2} \alpha^{2}}-k_{x}^{2}\right) \phi_{2}=0,
$$

where we have been using the identity $|\alpha|^{\prime} /|\alpha|=\alpha^{\prime} / \alpha$. Eq. (17) is necessarily self-adjoint, as it is the result of a variational principle [20], and gives rise to a classical SturmLiouville problem. With suitably chosen boundary conditions, its analytical solution can be expressed in terms of Bessel functions of the first and second kind [21]. However, this analytical solution is quite complicated and lengthy, and hence its evaluation is tedious. In contrast, the direct numerical integration of eq. (17) is easier and proves to be much more stable. 
Numerical discussion. - In acoustics, one usually measures or wishes to predict the sound pressure of a wavefront at a certain position and time. For Desloge's metrics, the acoustic pressure is determined by

$$
p=\rho_{0} \dot{\phi}=i \omega \rho_{0} A^{+} e^{i\left(\omega t-k_{x} x\right)} \phi_{2}(y),
$$

where we have used eqs. (14)-(16). The only unknown contribution arises from $\phi_{2}(y)$. For its numerical computation, we recast eq. (17) in the form of a system of ODEs of first order

$$
\begin{aligned}
& \psi_{1}^{\prime}=\psi_{2}, \\
& \psi_{2}^{\prime}=\left(k_{x}^{2}-\frac{\omega^{2}}{c^{2} \alpha^{2}}\right) \psi_{1}-\frac{\alpha^{\prime}}{\alpha} \psi_{2},
\end{aligned}
$$

subject to the suitably chosen initial conditions

$$
\psi(0)=\left(\begin{array}{l}
\psi_{1}(0) \\
\psi_{2}(0)
\end{array}\right)=\left(\begin{array}{l}
\phi_{2}(0) \\
\phi_{2}^{\prime}(0)
\end{array}\right)=\left(\begin{array}{l}
1 \\
0
\end{array}\right)
$$

As a double check, we have tackled this Cauchy boundaryvalue problem with two different classes of MATLAB solvers, namely the standard MATLAB solver ode45 and the more sophisticated SBVP package [22,23]. The basic algorithm for ode45 is a standard Runge-Kutta method, whereas in the SBVP algorithm a polynomial collocation method is implemented. For efficiency, the code is equipped with an adaptive mesh algorithm. SBVP was designed for ODEs with singularities of the first kind [22] in the differential operator, but it also can be used to solve regular ODEs. Setting the relative and absolute tolerances to $10^{-12}$, both solvers show excellent agreement in the following computations.

For all numerical estimates, we choose the following typical values for the parameters: $c=343 \mathrm{~m} / \mathrm{s}$ (the speed of sound in dry air at $20^{\circ} \mathrm{C}$ ) and $k_{x}=1$ (unitary wavelength in $x$ direction), and $\omega=3000 \mathrm{~Hz}$ (an upper value within the socalled voice frequency band). Furthermore, to make all effects discernible, we fix the value of the acceleration/gravitational field strength at about 1000 times Earth's surface gravity. We also consider up and downward orientation with respect to the $y$-direction, i.e. $g_{0}= \pm 9810 \mathrm{~m} / \mathrm{s}^{2}$.

Fig. 1 compares how $\phi_{2}$ depends on $y$ as predicted by the UAF and the UGF model, respectively. As expected, both frequency shifts coincide for small values of $y$. This is the region where the two frames are acoustically equivalent and also match with the original gravity analogue under similar conditions. Since $g_{0}>0$, this corresponds to a downward acceleration, $c f$. eq. (8), and the wavelength grows with height, or equivalently, the frequency reduces with height. Therefore, we recognize an acoustic redshift.

For large $y$, the predictions of both models deviate considerably, which is due to the distinct asymptotic behaviour of the governing differential equation, eq. (17). It is straightforward to check analytically that in this limit the UAF model yields simple oscillatory motion with a constant amplitude, whereas the UGF variant of eq. (17), with $\alpha^{\prime} / \alpha=g_{0} / c^{2}$, produces an exponentially growing amplitude.

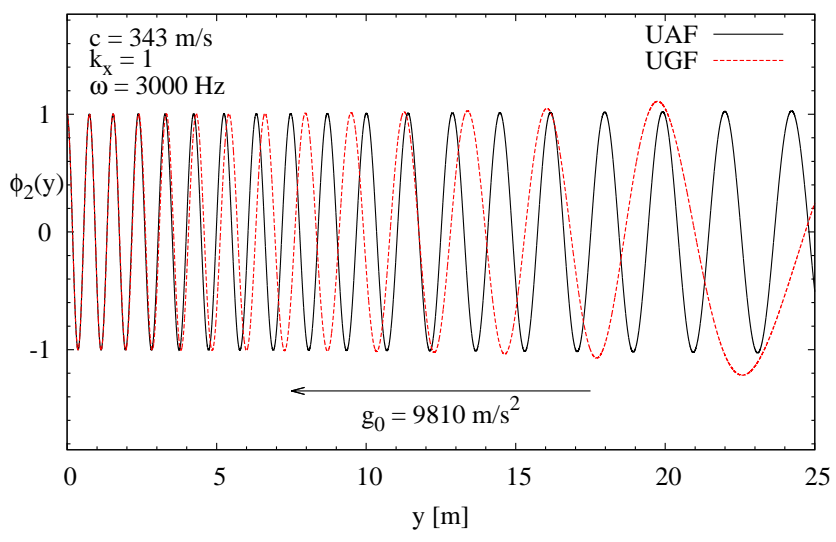

Fig. 1: The dependence of the acoustic potential $\phi_{2}$ on height $y$ for a uniformly accelerating frame (UAF) and in a uniform gravitational field (UGF). The acceleration $g_{0}>0$ corresponds to alignment in antiparallel $y$-direction. An acoustic redshift is observed.
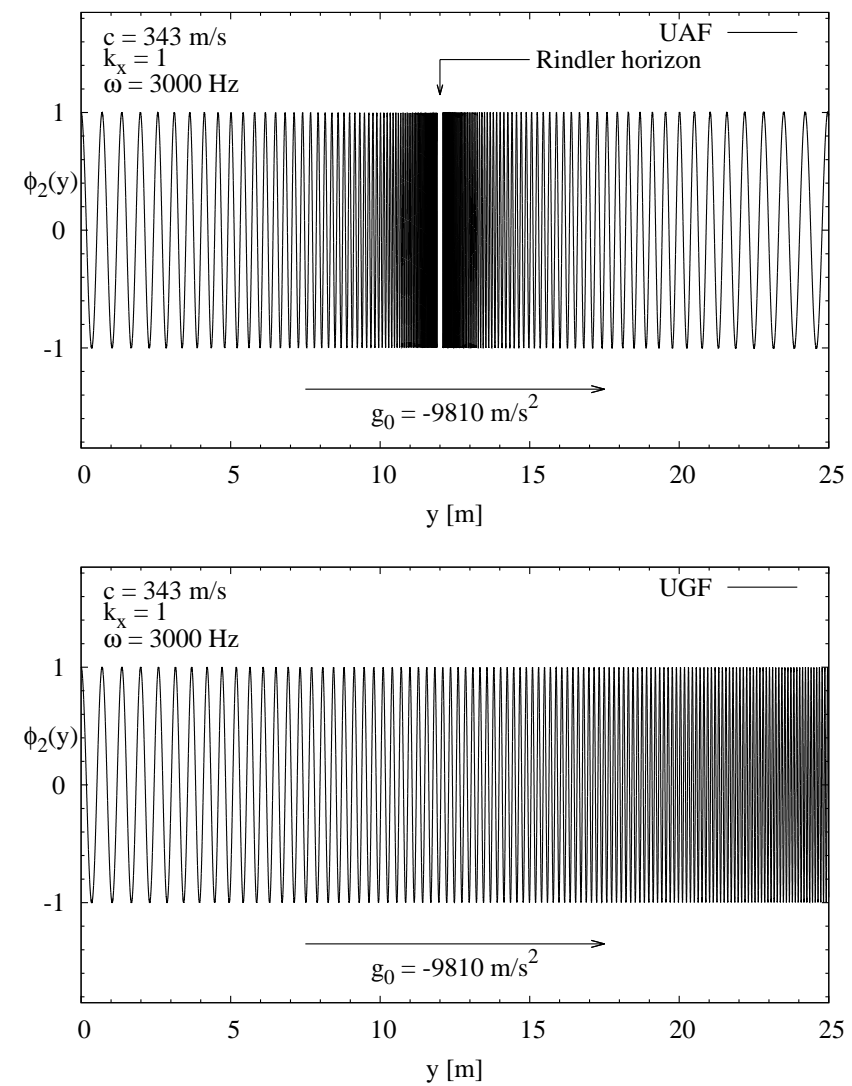

Fig. 2: The dependence of the acoustic potential $\phi_{2}$ on height $y$ with (a) uniform acceleration (UAF) and (b) uniform gravitational field (UGF). The acceleration $g_{0}<0$ corresponds to alignment along the $y$-direction. The UGF potential in (b) displays a flawless acoustic blueshift, whereas the UAF spacetime in (a) gives rise to a Rindler event horizon at $-g_{0} / c^{2} \approx 12 \mathrm{~m}$. 


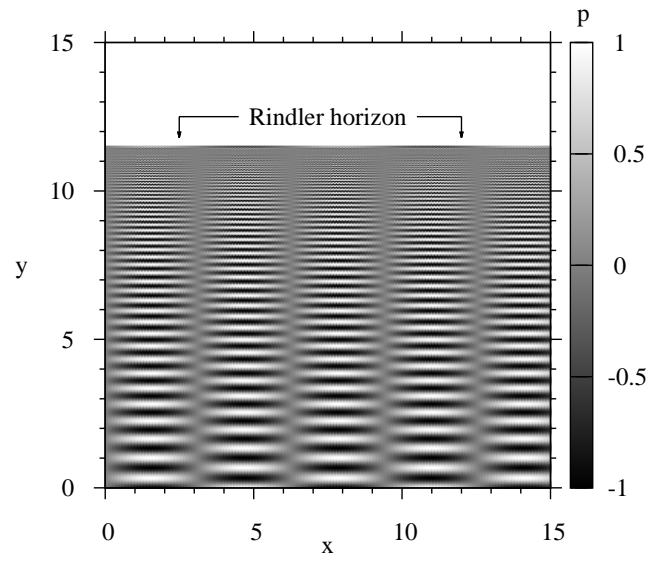

Fig. 3: A snapshot of the acoustic pressure $p$ at $t=0$ in the $x y$ plane with underlying UAF spacetime metric. The real part of eq. (18) is taken with $k_{x}=1$, and the total amplitude is normalized. The acoustic spacetime parameters in the plot are $g_{0}=-9810 \mathrm{~m} / \mathrm{s}^{2}$ and $c=343 \mathrm{~m} / \mathrm{s}$, which defines the Rindler horizon at $y_{0}=-c^{2} / g_{0} \approx$ $12 \mathrm{~m}$

In fig. 2, we study the UAF and UGF frequency shifts for an upward acceleration or gravitational field by choosing $g_{0}<0$. In the UGF case, the wavelength is rapidly decreasing which corresponds to the analogous effect of a wave moving from a higher to a lower gravitational potential. Hence, in fig. 2(b) an acoustic blueshift is observed.

For the UAF spacetime, the singularity of the underlying metric at $y_{0}=-c^{2} / g_{0}$ causes time to evolve slower in this region compared to a distant fixed observer. Time-dilation becomes ever more pronounced when approaching this region, until time freezes. Essentially, it takes infinite time to reach $y_{0}$ from both sides along the $y$-axis. This corresponds to a static acoustic barrier which the sound wave cannot surpass, and thus divides spacetime into two physical domains with no possible acoustic communication among them. Because of the symmetry $y \leftrightarrow-\left(y+2 c^{2} / g_{0}\right)$ under which the UAF acceleration reverses sign, the effect is also symmetric and identifies $y_{0}$ with the position of a symmetric Rindler event horizon. It is quite remarkable that this effect is independent of wave frequency or amplitude, as $y_{0}$ only depends on the acoustic spacetime properties $g_{0}$ and $c$.

Fig. 3 presents a full snapshot of the acoustic pressure $p$ at time $t=0$ calculated from the numerical values of $\phi_{2}$ for the UAF spacetime, $c f$. fig. 2(a). In fig. 3, the real part of eq. (18) is evaluated with unit wavelength $k_{x}=1$ and, for convenience, the resulting amplitude $\omega \rho_{0} A^{+}$is normalized. As before, the acoustic spacetime parameters are $g_{0}=-9810 \mathrm{~m} / \mathrm{s}^{2}$ and $c=343 \mathrm{~m} / \mathrm{s}$, which locates the Rindler event horizon on the positive $y$-axis at $y_{0}=-c^{2} / g_{0} \approx 12 \mathrm{~m}$. The incident pressure wave becomes extremely compressed when approaching the horizon (up to the point where the numerical simulation fails). Recall that this UAF event horizon corresponds to an underlying flat spacetime (viz. eq. (5)) and is an artifact of the coordinate choice alone. Nevertheless, its physical imple- mentation in an acoustic metamaterial has many challenging consequences.

In transformation optics, it has been argued that actual metamaterial losses in the laboratory setting would interfere with the genuine appearance of an event horizon. However, any attempt to compensate for these losses would also effectively produce Hawking radiation from the surface [24,25]. A similarly interesting phenomenon should be expected to emerge in transformation acoustics as well.

Conclusion. - In this work, we have applied a recently proposed covariant spacetime approach of metamaterial acoustics [12] for engineering an acoustic metadevice with wave properties that mimic gravitational frequency shifts. Two spacetimes are implemented and discussed, the flat UAF spacetime for a uniformly accelerating frame and the curved UGF spacetime representing a uniform gravitational field [13].

For both spacetimes, the constitutive relations among the scalar bulk modulus and the mass-density tensor are specified in order to establish the correspondence of the curvilinear coordinate transformations between associated physical and virtual acoustic space and their respective material properties.

For UGF spacetime, wave propagation in the corresponding prefabricated acoustic metamedium exhibits the expected frequency shifts, depending on whether sound travels in the same or opposite direction of the chosen acceleration or gravitational field.

In the case of the UAF metric framework, it is shown that the acoustic wave analogue necessarily incorporates a linear event horizon, i.e., a boundary in spacetime beyond which events cannot acoustically affect any outside observers. Due to the similarity of the Rindler horizon to the event horizon of a Schwarzschild black hole, intriguing phenomena such as acoustic Hawking radiation may be studied in detail.

Moreover, the extraordinary acoustic properties of the UAF and UGF analogues in metamaterial acoustics may lead the path to the design of acoustic industrial devices with promising applications in the near future.

$$
* * *
$$

M. M. T. wishes to thank Markus Schöbinger for an introduction to the SBVP MATLAB solver and acknowledges partial support by the Universidad Politécnica de Valencia (PAID-0012) and the International Office of the Vienna University of Technology.

\section{REFERENCES}

[1] Greenleaf A., Kurylev Y., Lassas M. and Uhlmann G., Phys. Rev. Lett., 99 (2007) 183901.

[2] Thompson R. T. and Frauendiener J., Phys. Rev. D, 82 (2010) 124021.

[3] Smolyaninov I. I., J. Opt., 13 (2011) 024004.

[4] Ginis V., Tassin P., Craps B. and Veretennicoff I., Opt. Express, 18 (2010) 5350.

[5] Norris A. N., J. Acoust. Soc. Am., 125 (2009) 839.

[6] Cummer S. A. and Schurig D., New J. Phys., 9 (2007) 45. 
[7] Chen H. Y. and Chan C. T., J. Phys. D, 43 (2010) 113001.

[8] Chen H. Y. and Chan C. T., Appl. Phys. Lett., 91 (2007) 183518.

[9] Post E. J., Formal Structure of Electromagnetics (NorthHolland, Amsterdam) 1962.

[10] Thompson R. T., Cummer S. A. and Frauendiener J., $J$. Opt., 13 (2011) 055105.

[11] García-Meca C. and Tung M. M., Math. Comput. Model., 57 (2013) 1773.

[12] Tung M. M., Europhys. Lett., 98 (2012) 34002.

[13] Desloge E. A., Am. J. Phys., 57(12) (1989) 1121.

[14] Desloge E. A., Am. J. Phys., 58(9) (1990) 856.

[15] Acedo L. and Tung M. M., Eur. J. Phys., 33 (2012) 1073.

[16] Muñoz G. and Jones P., Am. J. Phys., 78(4) (2010) 377.

[17] Calin O. and Der-Chen C., Geometric Mechanics on Riemannian Manifolds (Birkhäuser, Boston) 2005.

[18] Visser M., Barceló C. and Liberati S., Gen. Rel. Grav., 34 (2002) 1719.

[19] FliPPI P. et al., Acoustics: Basic Physics, Theory and Methods (Academic Press, San Diego) 1998, p. 21.

[20] Lanczos C., The Variational Principles of Mechanics (University of Toronto Press, Toronto) 1949, p. 301.

[21] Tung M. M. and Peinado J., in Progress in Industrial Mathematics at ECMI 2012, edited by FonTES M. et al., Series: Mathematics in Industry (Springer-Verlag, Berlin), in press.

[22] AuZinger W., Kneisl G., Koch O. and Weinmüller E., Numer. Algorithms, 33 (2003) 27.

[23] Auzinger W., Kneisl G., Koch O. and Weinmüller E., SBVP 1.0 - MaTLAB Solver for Singular Boundary Value Problems, available at http://www .math.tuwien.ac.at/ ewa/.

[24] Thompson R. T. and Frauendiener J., Phys. Rev. D, 82 (2010) 124021.

[25] Smolyaninov I. I., Hwang E. and Narimanov E., Phys. Rev. B, 85 (2012) 235122. 